\title{
System measure for persistence in holographic recording and application to singly-doped and doubly-doped lithium niobate
}

\author{
Ali Adibi, Karsten Buse, and Demetri Psaltis
}

\begin{abstract}
We define a measure for persistence in holographic recording. Using this measure and the known measures for dynamic range and sensitivity, we compare the performance of singly-doped and doublydoped $\mathrm{LiNbO}_{3}$ crystals. We show that the range of performance that can be obtained using doubly-doped crystals is much larger than that obtained using singly-doped ones. (C) 2001 Optical Society of America OCIS codes: $\quad 090.009,090.2900,090.7330,210.2860$.
\end{abstract}

\section{Introduction}

Several holographic storage demonstrations that use iron-doped photorefractive lithium niobate $\left(\mathrm{LiNbO}_{3}\right.$ : Fe) were presented in the past few years. ${ }^{1-3}$ Erasure of holograms during readout has been one of the major problems in the practical realization of holographic read-write memories. We recently proposed and demonstrated the two-center holographic recording method to solve this problem by using doubly-doped $\mathrm{LiNbO}_{3} \cdot{ }^{4,5}$ Several aspects of persistent holographic storage in doubly-doped $\mathrm{LiNbO}_{3}$ (i.e., hologram multiplexing, ${ }^{6}$ sensitivity improvement, ${ }^{7}$ optimization, ${ }^{5,8}$ and the role of different dopants $^{9-11}$ ) were investigated. In this paper we present a framework for the comparison of the performances of singly-doped and doubly-doped $\mathrm{LiNbO}_{3}$ crystals for holographic storage. By defining a new measure for persistence, we show that by going from singly-doped crystals to doubly-doped crystals, we add one degree of freedom in the optimization of the performance for holographic storage. We show that the range of performance characteristics that can be obtained using a doubly-doped crystal is much larger

A. Adibi (e-mail address: adibi@ee.gatech.edu) is with the School of Electrical and Computer Engineering, Georgia Institute of Technology, Atlanta, Georgia 30332. K. Buse is with the Physikalisches Institut, Universität Bonn, Wegelerstrasse 8, D-53115 Bonn, Germany. D. Psaltis is with the Department of Electrical Engineering, California Institute of Technology, Pasadena, California 91125.

Received 16 November 2000; revised manuscript received 29 May 2001.

0003-6935/01/295175-08\$15.00/0

(C) 2001 Optical Society of America than that obtained using a singly-doped one. We also explain the major trade-offs among dynamic range, sensitivity, and persistence in holographic storage in $\mathrm{LiNbO}_{3}$ crystals.

\section{Performance Measures in Holographic Recording}

The main performance measures in holographic recording are dynamic range ${ }^{12}(M / \#)$, sensitivity ${ }^{13}(S)$, and persistence. The measures for the dynamic range $(M / \#)$ and sensitivity $(S)$ are defined as

$$
\begin{aligned}
& M / \#=\frac{A_{0} \tau_{e}}{\tau_{r}}, \\
& S=\frac{\left.\frac{\mathrm{d} \sqrt{\eta}}{\mathrm{d} t}\right|_{t=0}}{I_{\operatorname{Rec}} L}=\frac{A_{0} / \tau_{r}}{I_{\operatorname{Rec}} L} .
\end{aligned}
$$

In these equations $A_{0}$ is the saturation hologram strength, $\eta$ is diffraction efficiency, and $\tau_{r}$ and $\tau_{e}$ are recording and erasure time constants during multiplexing holograms, respectively. Note that by using $\sqrt{\eta}=A$ (where $A$ is the hologram strength) we assume that $\eta \ll 1$, which is the case when we multiplex several holograms. In hologram multiplexing experiments, erasure of previously written holograms is caused by recording new holograms. The symbols $I_{\text {Rec }}$ and $L$ denote the total recording intensity and the thickness of the recording medium (or the hologram), respectively. If we multiplex $M$ holograms in the same volume appropriately, ${ }^{14}$ each will have a diffraction efficiency equal to $\eta=[(M /$ $\#) / M]^{2}$. Because $M / \#$ depends on crystal thickness 
$(L)$, we define a normalized dynamic range measure as

$$
M^{\prime} / \#=\frac{M / \#}{L},
$$

to normalize the effect of thickness similar to the normalization for $S$.

Although the performance measures for dynamic range and sensitivity are well defined, a quantitative measure for persistence is still missing. To define such a measure, we need to quantify the erasure of a hologram during readout. Such a measure should not depend on experimental conditions such as readout light intensity. One measure for persistence is the erasure time constant $\left(\tau_{e}^{\prime}\right)$ caused by the readout beam. However, $\tau_{e}^{\prime}$ is inversely proportional to the reading light intensity $\left(I_{\mathrm{Rd}}\right)$. Therefore we can define an intensity-independent measure for persistence as

$$
P=\tau_{e}^{\prime} I_{\mathrm{Rd}},
$$

where $P$ is in units of $\mathrm{J} / \mathrm{cm}^{2}$. It represents the reading energy density required for reducing the hologram strength (or $\sqrt{\eta}$ ) by a factor of $\exp (1)$. Note that $\tau_{e}^{\prime}$ in Eq. (4) is, in general, different from $\tau_{e}$ in Eq. (1). The former is the erasure time constant of a hologram during readout, whereas the latter is the erasure time constant of a hologram during the recording of other holograms in the same location. In this paper we assume that dark erasure mechanisms such as dark conductivity and ionic compensation in the dark are negligible compared with light-induced erasure.

The disadvantage of $P$ as a measure for persistence is that it does not incorporate the hologram strength. In other words, under similar conditions (same reading intensity and same $P$ ), we can read a strong hologram (for example, $\eta=10^{-2}$ ) for a longer time in comparison with a weak hologram (for example, $\eta=$ $10^{-4}$ ) before the diffraction efficiency reaches the minimum acceptable value (set by the noise level). Therefore we need to modify $P$ to incorporate the hologram strength. To define such a measure, we first define a reference storage system. We assume that such a system is composed of $M$ holograms multiplexed in the same volume and that the minimum acceptable diffraction efficiency of each reconstructed plane-wave hologram is $\eta_{\text {min }}$. We also assume that in such a system, we need to have $N_{\mathrm{ph}}$ photons for each pixel with area $A_{\text {pixel }}$ at the detector to obtain an acceptable signal-to-noise ratio. ${ }^{15}$ We define the measure for persistence, $R / \#$, as the number of times we can read the information in the entire module (all $M$ holograms) before the diffraction efficiency of each hologram falls below the minimum acceptable value $\left(\eta_{\min }\right)$.

The reading time of each hologram $\left(t_{h}\right)$ depends on the intensity of the reconstructed beam and the number of photons per pixel $\left(N_{\mathrm{ph}}\right)$ required to obtain an acceptable signal-to-noise ratio. The formula for $t_{h}$ can be written as

$$
t_{h}=\frac{N_{\mathrm{ph}} h v}{I_{\text {out }} A_{\text {pixel }}}=\frac{N_{\mathrm{ph}} h v}{\eta I_{\mathrm{Rd}} A_{\text {pixel }}},
$$

where $h v$ is the energy of a single photon at the reading light frequency and $\eta, I_{\text {out }}$, and $\mathscr{A}_{\text {pixel }}$ are the diffraction efficiency of each hologram, output (or reconstructed) intensity, and the area of each pixel, respectively. Note that $I_{\text {out }}, I_{\mathrm{Rd}}$, and $\mathscr{A}_{\text {pixel }}$ are defined in the same plane. In our calculations we use the values at the detector plane (usually each pixel of the detector is matched to one pixel of the spatial light modulator). However, we can use the values at any other plane, including the output of the recording medium (or hologram). The possible focusing of the recording and the reading beams changes $I_{\mathrm{Rd}}$ and $A_{\text {pixel }}$, but the product $I_{\mathrm{Rd}} \mathscr{A}_{\text {pixel }}$ (or $I_{\text {out }} \mathscr{A}_{\text {pixel }}$ ) will remain unaffected. The role of focusing a light beam is to reduce the cross-sectional area of the beam (and therefore $A_{\text {pixel }}$ ), which increases the intensity (power per unit area) of the beam accordingly. In other words, the reading time of each hologram depends on the number of photons per unit time available per pixel, and this is the same at the hologram and at the detector for the fixed reading power (even though the pixel sizes at these two planes are different).

During readout, the decay of a hologram with strength $\sqrt{\eta}=A$ and erasure time constant $\tau_{e}^{\prime}$ is represented by

$$
\frac{\mathrm{d} A}{\mathrm{~d} t}=-\frac{A}{\tau_{e}^{\prime}} .
$$

Therefore the number of times that we can read the entire memory module ( $M$ holograms) in a time $\mathrm{d} t$ is

$$
\frac{\mathrm{d} t}{M t_{h}}=-\frac{\tau_{e}^{\prime}}{M t_{h}} \frac{\mathrm{d} A}{A}=-\frac{\left(\tau_{e}^{\prime} I_{\mathrm{Rd}}\right) \mathscr{A}_{\mathrm{pixel}}}{M N_{\mathrm{ph}} h v} A \mathrm{~d} A,
$$

where we replaced $\mathrm{d} t=-\tau_{e}^{\prime} \mathrm{d} A / A$ from Eq. (6) and used the relation $\eta=A^{2}$ between diffraction efficiency and hologram strength. Using the initial and final hologram strengths $\left[A_{i}=\sqrt{\eta_{i}}=(M / \#) / M\right.$ and $A_{f}=\sqrt{\eta_{\text {min }}}$, respectively], we can integrate Eq. (7) to obtain

$$
\begin{aligned}
R / \# & \equiv \frac{\left(\tau_{e}^{\prime} I_{\mathrm{Rd}}\right) \mathscr{A}_{\text {pixel }}}{M N_{\mathrm{ph}} h v} \int_{\sqrt{\eta_{i}}}^{\sqrt{\eta_{\text {min }}}}(-A) \mathrm{d} A \\
& =\frac{\left(\tau_{e}^{\prime} I_{\mathrm{Rd}}\right) \mathscr{A}_{\text {pixel }}}{2 M N_{\mathrm{ph}} h v}\left[\left(\frac{M / \#}{M}\right)^{2}-\eta_{\text {min }}\right] \\
& =\frac{P A_{\text {pixel }}}{2 M N_{\mathrm{ph}} h v}\left[\left(\frac{M / \#}{M}\right)^{2}-\eta_{\text {min }}\right] .
\end{aligned}
$$

The $R / \#$ is the number of times a storage module of $M$ multiplexed holograms recorded in a material with a given $M / \#$ can be read before the diffraction efficiency of each hologram falls below the minimum acceptable value $\left(\eta_{\min }\right)$. To measure $R / \#$ experimentally, we compute $P=\tau_{e}^{\prime} I_{\mathrm{Rd}}$ from the erasure 
response of a hologram and put the computed value along with the previously measured $M / \#$ into Eq. (8).

\section{Performance Range in Singly-Doped and Doubly-Doped Crystals}

In singly-doped crystals, $\tau_{e} I_{\mathrm{Rec}}$ and $\tau_{e}^{\prime} I_{\mathrm{Rd}}$ are equal (or $\tau_{e}=\tau_{e}^{\prime}$ for equal recording and reading intensities) because the physical erasure mechanisms during recording and reading in such crystals are the same. As a result, in singly-doped crystals, $P$ (and therefore $R / \#)$ is totally dependent on $M / \#$ and $S$ as shown below. By combining Eqs. (1), (2), and (3), we obtain

$$
P=\frac{M / \#}{L S}=\frac{M^{\prime} / \#}{S} .
$$

This means that we can freely choose only two parameters from the trio of $M / \#, S$, and $R / \#$ in holographic recording in a singly-doped crystal. The simplified formulas for the variation of $M / \#$ and $S$ of a singly-doped $\mathrm{LiNbO}_{3}: \mathrm{Fe}$ crystal in the regime of the domination of bulk photovoltaic effect (for the case of congruently melting crystals) is ${ }^{16}$

$$
\begin{aligned}
M / \# & \propto \frac{\kappa_{\mathrm{Fe}} \gamma_{\mathrm{Fe}}}{q_{\mathrm{Fe}} s_{\mathrm{Fe}}}\left(N_{\mathrm{Fe}}-N_{\mathrm{Fe}}^{-}\right), \\
S & \propto \kappa_{\mathrm{Fe}} N_{\mathrm{Fe}}^{-},
\end{aligned}
$$

where $q_{\mathrm{Fe}} s_{\mathrm{Fe}}, \kappa_{\mathrm{Fe}}$, and $\gamma_{\mathrm{Fe}}$ are absorption cross sections for the excitation of electrons from the Fe traps to the conduction band (at the recording wavelength), the bulk photovoltaic constant of the Fe traps (at the recording wavelength), and the recombination coefficient of the $\mathrm{Fe}$ traps, respectively. Furthermore, $N_{\mathrm{Fe}}$ and $N_{\mathrm{Fe}}^{-}$are the total Fe concentration and the concentration of electrons in the Fe traps, respectively. Using Eq. (9) and expressions (10) and (11) we obtain

$$
P \propto \frac{\gamma_{\mathrm{Fe}}}{q_{\mathrm{Fe}} s_{\mathrm{Fe}}} \frac{N_{\mathrm{Fe}}-N_{\mathrm{Fe}}^{-}}{N_{\mathrm{Fe}}^{-}} .
$$

In expressions (10) and (11) we considered only the important parameters that depend on the photorefractive $(\mathrm{Fe})$ centers. In these equations we neglected the light absorption through the crystal. For considerable absorption, both $M / \#$ and $S$ are multiplied by $\exp (-\alpha L / 2)$, where $\alpha$ is the intensity absorption coefficient of the crystal at the recording wavelength. From these equations we can see that the trade-off between $M / \#$ and $S$ can be performed by changing $N_{\mathrm{Fe}}^{-}$, the electron concentration in the $\mathrm{Fe}$ traps. This can be performed by annealing (oxidation-reduction) of the crystal. However, the range of this trade-off is limited by the absorption of recording and reading light through the crystal. Increasing $N_{\mathrm{Fe}}^{-}$beyond some limit results in large absorption coefficient $\alpha$ reducing both $M / \#$ and $S$.

Another method for changing both $M / \#$ and $S$ is to change the other parameters $\left(q_{\mathrm{Fe}} s_{\mathrm{Fe}}, \kappa_{\mathrm{Fe}}\right.$, and $\left.\gamma_{\mathrm{Fe}}\right)$ in expressions (10) and (11). However, these parameters are the properties of the dopant, and they are (a)

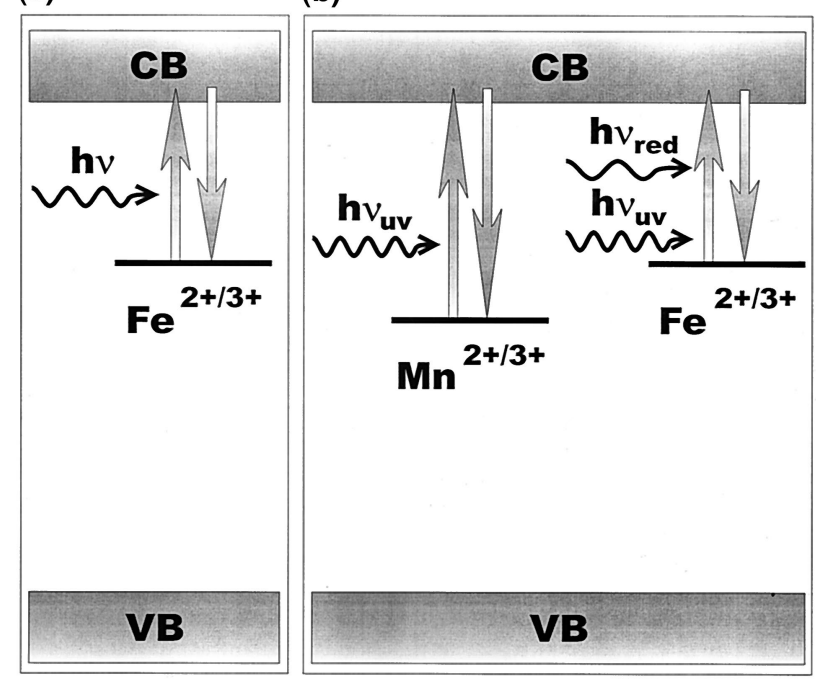

Fig. 1. Energy band diagram for a typical $\mathrm{LiNbO}_{3}$ crystal doped with (a) Fe and (b) Fe and Mn. CB, conduction band; VB, valance band.

fixed for a singly-doped crystal. We cannot change these parameters for a grown crystal by simple treatments like annealing. Therefore we need to change the dopant to change the parameters in expressions (10) and (11). However, the known effective photorefractive centers in $\mathrm{LiNbO}_{3}$ are limited to $\mathrm{Fe}, \mathrm{Mn}$, $\mathrm{Cu}$, and $\mathrm{Cr}$. Another idea to extend the range of obtainable $M / \#$ and $S$ is to use a doubly-doped crystal, for example, $\mathrm{LiNbO}_{3}: \mathrm{Fe}: \mathrm{Mn}$. Figure 1 compares the band diagram of a $\mathrm{LiNbO}_{3}: \mathrm{Fe}$ crystal with that of a $\mathrm{LiNbO}_{3}: \mathrm{Fe}: \mathrm{Mn}$ crystal. Because $\mathrm{Mn}$ traps are deeper than Fe traps, electrons occupy Mn traps before they occupy $\mathrm{Fe}$ traps. By highly oxidizing a $\mathrm{LiNbO}_{3}: \mathrm{Fe}: \mathrm{Mn}$ crystal, we can have all the Fe traps as well as most of the Mn traps empty. The remaining electrons occupy a small portion of the Mn traps, and the crystal properties are similar to those of a highly oxidized $\mathrm{LiNbO}_{3}: \mathrm{Mn}$ crystal. However, we can fill all Mn traps as well as a large portion of the Fe traps with electrons by highly reducing the crystal. Such a crystal can act like a highly reduced $\mathrm{LiNbO}_{3}: \mathrm{Fe}$ crystal. The properties of the $\mathrm{LiNbO}_{3}$ : Fe:Mn crystal at a different oxidation-reduction state is between the two extreme cases mentioned above (from highly oxidized $\mathrm{LiNbO}_{3}: \mathrm{Mn}$ to highly reduced $\mathrm{LiNbO}_{3}: \mathrm{Fe}$ ). Therefore we can generate an effective trap with tunable properties by using a doubly-doped crystal. In other words, depending on the doping concentrations and oxidation-reduction state, the combination of the Fe traps and the $\mathrm{Mn}$ traps can be considered as an effective set of traps with properties between those of $\mathrm{Fe}$ and Mn traps.

If we use the light of only one wavelength for holographic recording in a $\mathrm{LiNbO}_{3}: \mathrm{Fe}: \mathrm{Mn}$ crystal, the previous relation between $M / \#, S$, and $R / \#$ [Eq. (9)] still holds because the erasure mechanisms during readout and during recording of other holograms are the same. However, the range of obtainable $M / \#$ 
and $S$ is larger now due to the possibility of tuning the properties of the effective traps by annealing (oxidation-reduction). To have more control over $M / \#, S$, and $R / \#$ and to be able to adjust all three measures, instead of just two, independently, we can use two-center holographic recording in a doublydoped crystal. ${ }^{4}$ To use a $\mathrm{LiNbO}_{3}: \mathrm{Fe}: \mathrm{Mn}$ crystal for two-center recording, we first need to oxidize the crystal properly ${ }^{17}$ so as to have all Fe traps as well as a portion of the Mn traps initially empty. Two-center holographic recording is performed by a homogeneous UV beam for sensitization and two coherent beams (at a longer wavelength) for recording. The basic idea of two-center holographic recording is to bring with the UV light electrons from $\mathrm{Mn}$ to Fe via the conduction band, to use these electrons to record the hologram with red or green light, and eventually to transfer the electrons from the Fe centers back to the Mn centers by red or green light. This results in a hologram stored in Mn centers that persists against further red or green illumination.

The unique property of two-center holographic recording is the distinction between the erasure time constant of a hologram during the recording of other holograms $\left(\tau_{e}\right)$ and the erasure time constant during readout of a hologram $\left(\tau_{e}^{\prime}\right)$. The erasure of a hologram during the recording of other holograms is caused by the simultaneous presence of sensitizing (UV) and recording (red or green) beams. In contrast, erasure of a hologram during readout is caused only by the reading (red or green) beam because the sensitizing (UV) beam is not present during readout. Furthermore, the electron concentration in the shallower $(\mathrm{Fe})$ traps is considerable during recording (owing to the sensitization process), whereas it is negligible (practically zero) during readout (because the hologram is recorded in the Mn traps). Therefore the erasure during readout is much weaker than that during the recording of other holograms $\left(\tau_{e}^{\prime}\right.$ is much larger than $\tau_{e}$ ) in two-center recording in a doubly-doped crystal. Furthermore, $\tau_{e}^{\prime}$ and $\tau_{e}$ are not proportional to each other, and Eq. (9) is not applicable to two-center recording. Therefore we can trade off all three of $M / \#, S$, and $R / \#$ in the two-center recording instead of two $(M / \#$ and $S)$ in normal recording. In other words, by using a doubly-doped crystal with two-center recording we can add an additional degree of freedom (persistence) to our design.

\section{Experiments}

To verify the above-mentioned claims, we performed holographic recording and readout experiments with two $0.85-\mathrm{mm}$-thick $\mathrm{LiNbO}_{3}: \mathrm{Fe}: \mathrm{Mn}$ crystals from the same boule, each doped with 0.075 wt. $\% \mathrm{Fe}_{2} \mathrm{O}_{3}$ and 0.01 wt. \% $\mathrm{MnO}$. Both crystals were first oxidized for $1 \mathrm{~h}$ at $1000{ }^{\circ} \mathrm{C}$ in $\mathrm{O}_{2}$ atmosphere. The crystals were then reduced at $800{ }^{\circ} \mathrm{C}$ in $\mathrm{Ar}$ atmosphere for either one hour (XTAL1) or four hours (XTAL2). All Fe traps in XTAL1 are empty, whereas more than $90 \%$ of the Mn traps in this crystal are occupied by electrons. However, all Mn traps as well as a portion of Fe traps

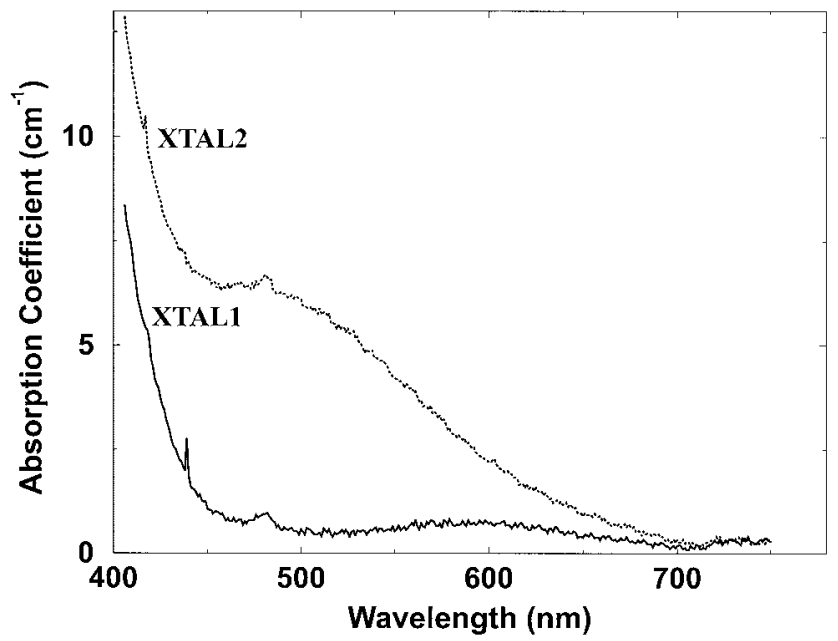

Fig. 2. Absorption spectra of the two crystals (XTAL1: oxidized, XTAL2: highly reduced) used in the holographic recording experiments.

are initially occupied by electrons in XTAL2. With this annealing treatment XTAL1 is appropriate for persistent holographic recording (two-center recording), and XTAL2 is good for normal singlewavelength recording with destructive readout. Figure 2 shows the absorption spectra of the two crystals. Normal holographic recording (no sensitizing light present) was performed with two plane waves with equal intensities and ordinary polarization, and two-center recording (sensitizing light present) was performed by similar recording beams and a homogeneous sensitizing (UV) beam. All recording experiments were performed with symmetric transmission geometry with the angle between the two recording beams outside the crystal of $42^{\circ}$. Ordinary polarization was used for both the recording beams as well as the reading beam.

Figure 3 shows typical recording and readout curves for four different recording strategies in $\mathrm{LiNbO}_{3}: \mathrm{Fe}: \mathrm{Mn}$. Normal holographic recording was used for the experiment corresponding to Figs. 3(a) and 3(b), and two-center recording was used for the other two cases [Figs. 3(c) and 3(d)]. We used the highly reduced crystal (XTAL2) for the experiment corresponding to Fig. 3(a) and the oxidized crystal (XTAL1) for the other three cases. The details of the experiments are summarized in the captions of Fig. 3.

The computed values for the best obtainable $M^{\prime} / \#$, $S$, and $R / \#$ for the four cases shown in Fig. 3 are summarized in Table 1. In these computations we assumed that the reference storage system is composed of $M=1000$ holograms multiplexed in the same volume of $1 \mathrm{~cm} \times 1 \mathrm{~cm} \times 1 \mathrm{~cm}$, where the minimum acceptable diffraction efficiency of each reconstructed plane-wave hologram is $\eta_{\min }=5 \times 10^{-6}$. We also assume that in such a system we need to have $N_{\mathrm{ph}}=1000$ photons for each $10 \mu \mathrm{m} \times 10 \mu \mathrm{m}$ pixel $\left(\mathscr{A}_{\text {pixel }}=10^{-6} \mathrm{~cm}^{2}\right)$ at the detector to obtain an acceptable signal-to-noise ratio. To compute the values shown in Table 1, we performed several experi- 
(a)

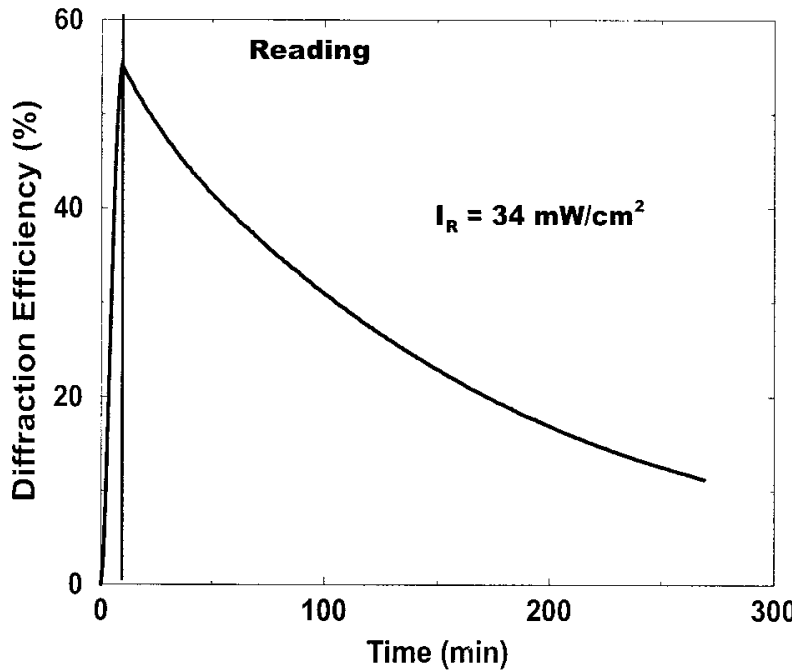

(c)

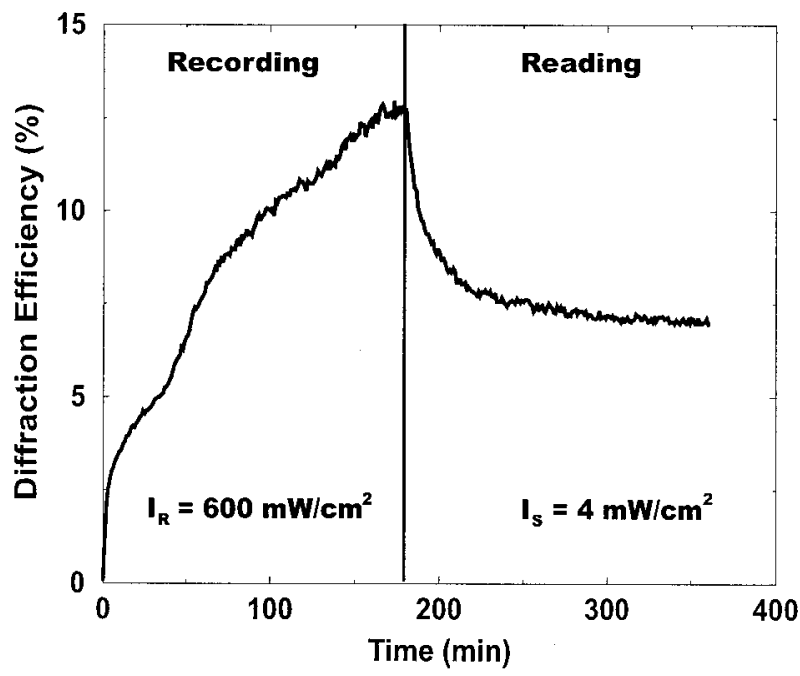

(b)

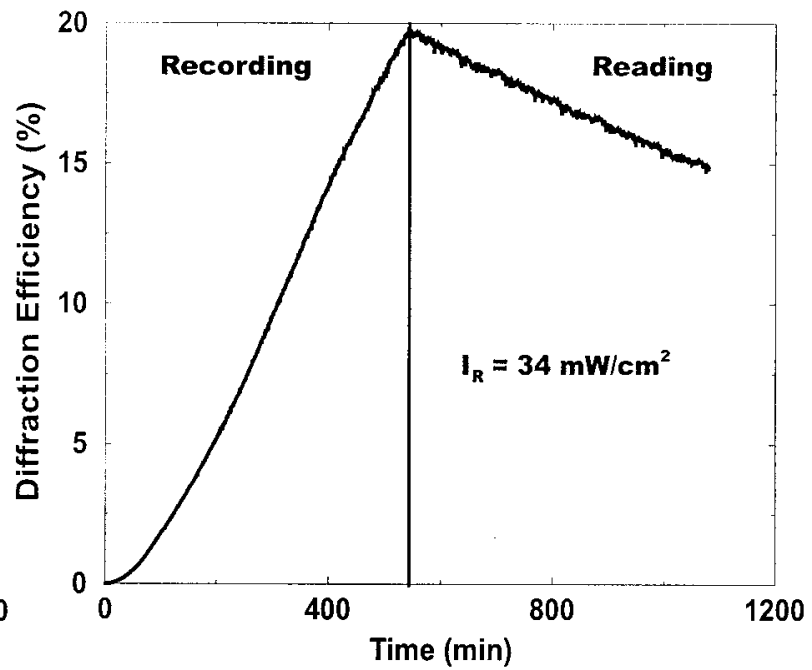

(d)

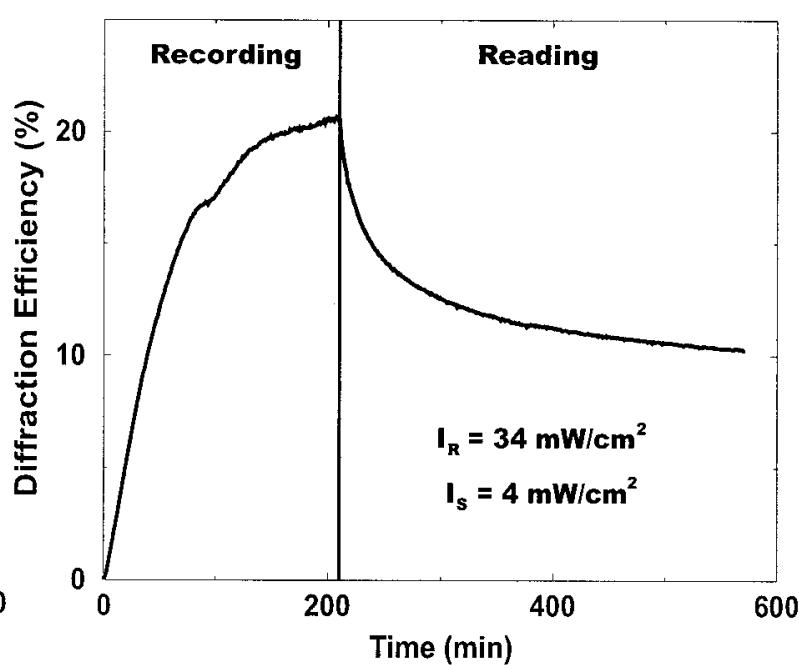

Fig. 3. Recording and readout curves for a plane-wave hologram in a 0.85-mm-thick $\mathrm{LiNbO}_{3}: \mathrm{Fe}: \mathrm{Mn}$ crystal. (a) Recording with two plane waves (wavelength of $488 \mathrm{~nm}$, intensity of each beam $17 \mathrm{~mW} / \mathrm{cm}^{2}$, ordinary polarization) in the highly reduced sample XTAL2. (b) Recording with two plane waves (transmission geometry, wavelength of $514 \mathrm{~nm}$, intensity of each beam $17 \mathrm{~mW} / \mathrm{cm}^{2}$, ordinary polarization) in the oxidized sample XTAL1. (c) Recording with two plane waves (transmission geometry, wavelength of $633 \mathrm{~nm}$, intensity of each beam $300 \mathrm{~mW} / \mathrm{cm}^{2}$, ordinary polarization) and one sensitizing beam in XTAL1. (d) Recording with two plane waves (transmission geometry, wavelength of $514 \mathrm{~nm}$, intensity of each beam $17 \mathrm{~mW} / \mathrm{cm}^{2}$, ordinary polarization) and one sensitizing beam in XTAL1. Total recording and sensitizing intensities ( $I_{R}$ and $I_{S}$, respectively) are shown in the figures. The intensity of the reading beam during readout in each case is half the corresponding recording intensity. The homogeneous sensitizing beams in both (c) and (d) were from a 100-W UV lamp (wavelength of $404 \mathrm{~nm}$, intensity $4 \mathrm{~mW} / \mathrm{cm}^{2}$ ). Erasure of a hologram in each case was performed by one of the recording beams rotated to result in Bragg-mismatch erasure.

ments for each case and averaged the values obtained from the individual experiments. In computing $M / \#$ and $S$, we assumed extraordinary polarization for the recording and readout light. This causes both $M / \#$ and $S$ to increase by a factor of approximately $r_{33} / r_{13} \simeq$ 3 owing to the larger electro-optic coefficient of $\mathrm{LiNbO}_{3}$ for extraordinary polarization. Here $r_{33}$ and $r_{13}$ are the corresponding electro-optic coefficients of $\mathrm{LiNbO}_{3}$ for extraordinary and ordinary polarizations of the recording (and readout) beam, respectively. To include the effect of partial erasure during readout in the sensitivity of two-center recording, we multiplied the value of $S$ calculated from Eq. (2) by the ratio of the final hologram strength (after sufficient readout) to the saturation hologram strength (before any readout), as explained in Ref. 7 . The last row in Table 1 comments about the strength of holographic scattering and fanning as the sources for deterioration of the holograms especially during readout. Table 1 reveals the large range of performance that is obtained with a $\mathrm{LiNbO}_{3}: \mathrm{Fe}: \mathrm{Mn}$ crystal (note that XTAL1 and XTAL2 can be considered as one crystal in that they 
Table 1. Comparison of the Performance Measures of Different Recording Schemes in a LiNbO ${ }_{3}: \mathrm{Fe}_{\mathrm{Mn} \mathrm{Crystal}^{a}}$

\begin{tabular}{lllll}
\hline \multicolumn{1}{c}{ Recording Scheme } & Normal & Normal & Two-Center & Two-Center \\
\hline Crystal & XTAL2 & XTAL1 & XTAL1 & XTAL1 \\
Annealing & reduced & oxidized & oxidized & oxidized \\
Sensitizing wavelength $(\mathrm{nm})$ & - & - & 404 & 404 \\
Sensitizing intensity $\left(\mathrm{mW} / \mathrm{cm}^{2}\right)$ & - & 514 & 633 & 4 \\
Recording wavelength $(\mathrm{nm})$ & 488 & 34 & 600 & 314 \\
Recording intensity $\left(\mathrm{mW} / \mathrm{cm}^{2}\right)$ & 34 & 55 & 8 & 34 \\
$(M / \#) / L\left(\mathrm{~cm}^{-1}\right)$ & 100 & 0.018 & 0.01 & 0.2 \\
$S(\mathrm{~cm} / \mathrm{J})$ & 0.8 & 12 & 90 & 0.5 \\
$R / \#\left(10^{6}\right)$ & 1.5 & small & very small & small \\
Fanning & severe & low & very low & low \\
Absorption of recording beams & high & & \\
\hline
\end{tabular}

${ }^{a}$ The persistence measure $(R / \#)$ is calculated with Eq. (8), where the parameters of the reference system are $M=1000, N_{\mathrm{ph}}=1000$, $A_{\text {pixel }}=10^{-6} \mathrm{~cm}^{2}(10 \mu \mathrm{m} \times 10 \mu \mathrm{m}$ pixels $)$, and $\eta_{\min }=5 \times 10^{-6}$.

differ only in the oxidation-reduction state). Such a large range of performance cannot be obtained with a singly-doped $\mathrm{LiNbO}_{3}$ crystal.

From the numbers for $M / \#, S$, and $R / \#$ in Table 1, it seems that normal recording in the highly reduced crystal, XTAL2 [Fig. 3(a) or the second column in Table 1] has the best overall performance. However, fanning and absorption of recording and reading light are severe in this case. Strong fanning in the reduced sample XTAL2 results in the fast deterioration of the stored information. Strong absorption causes high reduction of $M / \#, S$, and $R / \#$ when a thick crystal (for example, $1 \mathrm{~cm}$ thick) is used. Oxidizing of the crystal as well as use of a longer recording wavelength ( $514 \mathrm{~nm}$ or $633 \mathrm{~nm}$ instead of $488 \mathrm{~nm}$ ) reduces both fanning and absorption and increases $R / \#$. However, they both reduce the sensitivity considerably, as shown in the third column of Table 1 [or Fig. 3(b)]. Both fanning and absorption are further reduced by use of two-center recording with UV and red in the oxidized crystal. Use of two-center recording with UV and red also further improves $R / \#$. However, sensitivity is very low in two-center recording with UV and red, as shown in the fourth column of Table 1 and Fig. 3(c). The very low sensitivity of the two cases shown in Figs. 3(b) and 3(c) is a major practical disadvantage. From the four cases shown in Fig. 3 and Table 1, the last one, i.e., two-center recording with UV and green in the oxidized crystal [Fig. 3(d) or the fifth column in Table 1] has the best overall performance. Although both fanning and absorption of recording light are weak in this case, two-center recording with UV and green offers good $M / \#$ and $S$ along with acceptable $R / \#$ for most practical purposes. The combination of good performance measures in this case is due to the large range of obtainable performance offered by the use of doubly-doped crystals in holographic recording.

\section{Discussion}

The wide range of performance characteristics shown in Fig. 3 is interesting. It is important to note that the crystals used in all four cases depicted in Fig. 3 are essentially the same as the only difference is their oxidation-reduction state. Therefore the results summarized in Table 1 specify the range of performance that can be obtained in a doubly-doped crystal. Figure 3(a) shows that recording in a reduced crystal is fast and strong, but readout erases the hologram very fast. Strong holograms can be recorded with one wavelength (normal recording) in the more oxidized crystal (XTAL1), but recording is very slow. The holograms recorded in such a crystal have good persistence as they can be readout for a long time before their diffraction efficiency drops too much [Fig. $3(\mathrm{~b})$. Figures 3(c) and 3(d) show that by adding one homogeneous sensitizing beam to the recording system and using the more oxidized crystal (XTAL1) we can add another dimension to the performance characteristics. This added dimension is persistence. Recording with red light has better persistence than recording with green light; sensitivity is much better in recording with green light. This means that we can improve sensitivity by sacrificing some persistence. This range of performance characteristics obtained by use of a doubly-doped $\mathrm{LiNbO}_{3}$ crystal cannot be obtained by use of a singly-doped crystal by any means. For example, the range of performance in normal recording in a $\mathrm{LiNbO}_{3}: \mathrm{Fe}: \mathrm{Mn}$ crystal covers from that of a highly oxidized $\mathrm{LiNbO}_{3}: \mathrm{Mn}$ crystal to that of a highly reduced $\mathrm{LiNbO}_{3}: \mathrm{Fe}$ crystal. Furthermore, we can have persistence (or $R / \#$ ) as an independent measure by bringing in a sensitizing beam. The range of performance that can be obtained in a $\mathrm{LiNbO}_{3}: \mathrm{Fe}$ crystal covers only from that of a highly oxidized $\mathrm{LiNbO}_{3}: \mathrm{Fe}$ crystal to that of a highly reduced $\mathrm{LiNbO}_{3}: \mathrm{Fe}$ crystal.

It is evident from Table 1 that by adding long-term persistence through using two-center recording we sacrifice both $M / \#$ and $S$. One obvious reason for this loss in $M / \#$ and $S$ is that two-center recording has an extra step of bringing electrons from the deeper traps $(\mathrm{Mn})$ to the shallower ones $(\mathrm{Fe})$. This extra step reduces the recording speed compared with normal recording in singly-doped crystals in which we directly record from the shallower traps. The presence of the sensitizing UV beam also decreases the modulation depth for the electron concen- 
tration in the conduction band, resulting in a smaller $M / \#$. There is another important reason for losing $M / \#$ and $S$ in two-center holographic recording in $\mathrm{LiNbO}_{3}: \mathrm{Fe}: \mathrm{Mn}$ crystals: To obtain the best persistence we need to choose a recording wavelength large enough to suppress the erasure of the hologram recorded in the deeper traps during readout. Therefore we need to use red light for recording in $\mathrm{LiNbO}_{3}$ : Fe:Mn crystals. However, red light is not the best wavelength for recording from $\mathrm{Fe}$ traps in $\mathrm{LiNbO}_{3}$. The smaller absorption cross section of the Fe traps at $633 \mathrm{~nm}$ compared with that at $488 \mathrm{~nm}$ (that is a more appropriate wavelength for recording holograms from $\mathrm{Fe}$ traps in $\mathrm{LiNbO}_{3}$ ) results in a big loss in $S$. The photovoltaic coefficient of Fe traps at 633 $\mathrm{nm}$ is also much smaller than that at $488 \mathrm{~nm}$ (Ref. 18 and 19), resulting in losing $M / \#$ too. This extra loss due to the inefficiency of recording wavelength is the major source of loss in $M / \#$ and $S$ in two-center holographic recording with red and UV in $\mathrm{LiNbO}_{3}$ : Fe:Mn crystals. These extra losses can be avoided by use of better dopants or even better materials.

As seen from Table 1 , we can obtain huge $R / \#$ (approximately 100 millions) if we sacrifice sensitivity considerably. However, we may not need such huge values of $R / \#$ in practical application. Even if we can completely avoid erasure of the holograms during readout, dark erasure mechanisms cause the decay of the stored information. Therefore the recorded holograms need to be refreshed from time to time (for example, every few months). As a result, $R / \#$ needs to be large enough to ensure that the diffraction efficiency of each hologram does not fall below the minimum acceptable value before the refreshing time. A reasonable value for such requirement would be 0.5 million.

An interesting property of two-center recording is the relative insensitivity to fanning and holographic scattering. During recording, the presence of homogeneous sensitizing (UV) light prevents the build up of scattering holograms. During readout, the insensitivity of the deeper $(\mathrm{Mn})$ traps to the readout light is the reason for weak fanning.

The major challenge in designing a holographic storage system is the trade-offs among $M / \#, S$, and $R / \#$ along with the qualitative measures for absorption and fanning. Depending on the application, the main concern might be a subset of these measures. For example, if we do not want to write new information frequently but we need to read the information a lot, we need to use a recording scheme with large $R / \#$ and we can even sacrifice $S$ to obtain better $R / \#$. In such a case, we might use two-center holographic recording with red and UV in a properly oxidized $\mathrm{LiNbO}_{3}: \mathrm{Fe}: \mathrm{Mn}$ crystal [Fig. 3(c)]. If we need larger $M / \#$ than that obtained in two-center recording with red and UV, we can sacrifice some persistence $(R / \#)$ for $M / \#$ by using two-center holographic recording with green and UV [Fig. 3(d)].

It is important to note that the experimental results summarized in Table 1 were obtained by recording plane-wave holograms in transmission geometry with ordinary polarization for the recording beams. We also normalized the results for extraordinary polarization to obtain the best achievable values of the performance measures. In an actual system, we record holograms of two-dimensional data pages. The $M$ /\# for recording data pages is usually smaller than that for recording plane-wave holograms. Although there is no extensive quantitative study of this effect, reduction by a factor of 1.5-2 might be expected. Furthermore, if recording is performed with 90-deg geometry (instead of transmission geometry), further losses in $M / \#$ and $S$ is expected. A portion of this loss is due to the smaller grating period in 90-deg geometry, and a portion of it (a factor of approximately 3 ) is due to the requirement of the use of ordinary polarization in the 90-deg geometry. In all these cases, the reduction of either $M / \#$ or $S$ is similar for both singly-doped and doubly-doped crystals. Therefore our conclusion about the larger performance range in doubly-doped crystals is unaffected.

\section{Conclusion}

In conclusion we showed that the performance range that can be obtained by using a doubly-doped $\mathrm{LiNbO}_{3}$ crystal is much broader than that obtained by using a singly-doped crystal even if recording is performed by only two recording beams without sensitizing light (normal recording). We also showed that by adding a sensitizing beam to the holographic recording system (two-center recording), we can add one dimension for independent performance characteristics (persistence) that further broadens the range of performance characteristics that can be obtained. Furthermore, we defined for the first time a quantitative measure for persistence $(R / \#)$ in holographic recording and explained its relation with the other two major quantitative measures $M / \#$ and $S$. The main challenges in holographic recording are the trade-offs among $M / \#, S, R / \#$ and qualitative measures like fanning and absorption. Our results show that twocenter holographic recording with UV (wavelength $404 \mathrm{~nm}$ ) and green (wavelength $514 \mathrm{~nm}$ ) has the best overall performance for practical read-write holographic memory systems. Using this recording scheme in an appropriately annealed $1 \mathrm{~cm} \times 1 \mathrm{~cm} \times$ $1 \mathrm{~cm}$ crystal, we could expect to have $M / \# \simeq 10, S \simeq$ $0.2 \mathrm{~cm} / \mathrm{J}, R / \# \simeq 0.5$ millions, as well as weak fanning and light absorption during readout.

This research was supported by the U.S. Air Force Office of Scientific Research and by Air Force/Rome Lab award F0060297C0049. Support by the Deutscher Akademischer Austauschidienst/National Science Foundation is gratefully acknowledged.

\section{References}

1. F. H. Mok, "Angle-multiplexed storage of 5000 holograms in lithium niobate," Opt. Lett. 18, 915-917 (1993).

2. I. McMichael, W. Christian, D. Pletcher, T. Y. Chang, and J. H. Hong, "Compact holographic storage demonstrator with rapid access," Appl. Opt. 35, 2375-2379 (1996).

3. J. Ashley, M.-P. Bernal, M. Blaum, G. W. Burr, H. Coufal, R. K. 
Grygier, H. Günter, J. A. Hoffnagle, C. M. Jefferson, R. M. MacFarlane, B. Marcus, R. M. Shelby, G. T. Sincerbox, and G. Wittmann, "Holographic storage promises high data density," Laser Focus World 32, 81-93 (November 1996).

4. K. Buse, A. Adibi, and D. Psaltis, "Non-volatile holographic storage in doubly doped lithium niobate crystals," Nature 393, 665-668 (1998).

5. A. Adibi, K. Buse, and D. Psaltis, "Two-center holographic recording," J. Opt. Soc. Am. B 18, 584-601 (2001).

6. A. Adibi, K. Buse, and D. Psaltis, "Multiplexing holograms in $\mathrm{LiNbO}_{3}: \mathrm{Fe}: \mathrm{Mn}$ crystals," Opt. Lett. 24, 652-654 (1999).

7. A. Adibi, K. Buse, and D. Psaltis, "Sensitivity improvement in two-center holographic recording," Opt. Lett. 25, 539-541 (2000).

8. Y. W. Liu, L. R. Liu, and C. H. Zhou, "Prescription for optimizing holograms in LiNbO3:Fe:Mn," Opt. Lett. 25, 551-553 (2000).

9. X. F. Yue, A. Adibi, T. Hudson, K. Buse, and D. Psaltis, "Role of cerium in lithium niobate for holographic recording," J. Appl. Phys. 87, 4051-4055 (2000).

10. Y. W. Liu, L. R. Liu, C. H. Zhou, and L. Y. Xu, "Nonvolatile photorefractive holograms in $\mathrm{LiNbO}_{3}: \mathrm{Cu}: \mathrm{Ce}$ crystals," Opt. Lett. 25, 908-910 (2000).

11. Y. W. Liu, L. R. Liu, L. Y. Xu, and C. H. Zhou, "Experimental study of non-volatile holographic storage in doubly- and triplydoped lithium niobate crystals," Opt. Commun. 181, 47-52 (2000).

12. F. H. Mok, G. W. Burr, and D. Psaltis, "System metric for holographic memory systems," Opt. Lett. 21, 896-898 (1996).

13. P. Günter and J.-P. Huignard, eds., Photorefractive Materials and Their Applications I, Vol. 61, Topics in Applied Physics (Springer-Verlag, Berlin, 1988), pp. 47-52.

14. D. Psaltis, D. Brady, and K. Wagner, "Adaptive optical networks using photorefractive crystals," Appl. Opt. 27, 17521759 (1988).

15. E. Chuang, W. Liu, J. J. P. Drolet, and D. Psaltis, "Holographic random access memory (HRAM)," Proc. IEEE 87, 1931-1940 (1999).

16. K. Peithmann, A. Wiebrock, and K. Buse, "Photorefractive properties of highly doped lithium niobate crystals in the visible and near-infrared," Appl. Phys. B 68, 777-784 (1999).

17. A. Adibi, K. Buse, and D. Psaltis, "Effect of annealing in twocenter holographic recording," Appl. Phys. Lett. 74, 3767-3769 (1999).

18. E. Krätzig and H. Kurz, "Photo-induced currents and voltages in $\mathrm{LiNbO}_{3}$," Ferroelectrics 13, 295-296 (1976).

19. E. Krätzig and H. Kurz, "Photorefractive and photovoltaic effects in doped $\mathrm{LiNbO}_{3}$," Opt. Acta 24, 475-482 (1977). 